\section{CSL Behring vergibt Start-up-Jahres- stipendien für die nächste Generation von Gerinnungsforschern}

CSL Behring, ein weltweit führendes Unternehmen der Protein-Biotherapeutika-Industrie, gab beim XXI. Kongress der International Society on Thrombosis and Haemostasis (ISTH) bekannt, dass es 5 Jahresstipendien vergeben wird, um neue Forschungsprojekte auf dem Gebiet der Gerinnung zu unterstützen. Die Start-up-Stipendien von jeweils 20000 EUR (26780 USD) werden auf der Grundlage des wissenschaftlichen Verdiensts ihrer Arbeit an junge medizinische Forscher für präklinische und/oder klinische Projekte vergeben.

Das Stipendium wird nach Professor Norbert Heimburger benannt, einem Pionier der modernen Gerinnungstherapie, der über drei Jahr- zehnte lang Mitarbeiter von CSL Behring war. $\mathrm{Zu}$ seinen zahlreichen Leistungen zählt die Entwicklung virussicherer Plasmaprodukte auf Basis der Pasteurisierung. Dank seiner Forschungsaktivitäten konnte Behring 1981 das erste wirksam virusinaktivierte FVIII-Konzentrat auf den Markt bringen.

«CSL Behring setzt sich für die Förderung von Forschung ein, die unser Verständnis der Gerinnung voranbringt und die Behandlung von Gerinnungsstörungen wie der Hämophilie und des Von-Willebrand-Syndroms verbessert», sagte Roland Martin, der Leiter von Research and Development bei CSL Behring. «Es ist wichtig, die nächste Generation von Spezialisten dabei zu unterstützen, sich medizinisch und wissenschaftlich zu etablieren, um ständig neue Fortschritte auf diesem Indikationsgebiet zu erreichen.»
Bewerbungsschluss für die Stipendien ist der 1. Februar 2008. Die Bewerbungen werden von einem speziellen Auswahlkomitee begutachtet, dem vier auf dem Gebiet der Koagulation wissenschaftlich anerkannte Kliniker angehören.

Detailinformationen über das ProfessorNorbert-Heimburger-Stipendium und spezifische Kriterien für Bewerbungen unter: www.cslbehring.com/ProfHeimburgerAward.

Weitere Informationen bei

CSL Behring

Biotherapies for Life

Dr. Lutz Bonacker

Postfach 1230

35002 Marburg

Tel. +49 6421 39-4453,Fax -4146

lutz.bonacker@cslbehring.com

\section{Epoetin beta einmal wöchentlich therapiert Anämie bei onkologischen Patienten wirksam und sicher}

Bis zu $97 \%$ aller Tumorpatienten, die eine Chemotherapie erhalten, leiden unter Anämie. Sie gilt als eine der Hauptursachen für Fatigue, einen quälenden Erschöpfungszustand, der die Lebensqualität der Betroffenen stark einschränkt. Die enge Korrelation zwischen dem $\mathrm{Hb}$-Wert und der Lebensqualität der Betroffenen konnte in verschiedenen Studien nachgewiesen werden. Der Anstieg des Hb-Wertes hat einen positiven Effekt auf das Allgemeinbefinden der Betroffenen, wobei der stärkste Effekt auf die Lebensqualität bei einer Steigerung des $\mathrm{Hb}$-Wertes von 10 auf $12 \mathrm{~g} / \mathrm{dl}$ erzielt wird. Eine dauerhafte Steigerung des $\mathrm{Hb}$-Wertes kann durch die Gabe von rekombinanten Erythropoietinen, z.B. 30000 IE Epoetin beta (NeoRecormon ${ }^{\circledR}$ ), erreicht werden. Die Behandlung sollte laut Leitlinien der EORTC (European Organisation for Research and Treatment of Cancer) bei Krebspatienten mit Chemotherapie ab einem $\mathrm{Hb}-$ Wert von $9-11 \mathrm{~g} / \mathrm{dl}$ begonnen und bis zu einem Zielwert von 12-13 g/dl fortgesetzt werden. Ziel ist eine Verbesserung der Lebensqualität sowie die Vermeidung von Bluttransfusionen, die lediglich eine kurzfristige Korrektur der Anämie bewirken und mit einem erhöhten Risiko für Infektionen oder allergische Reaktionen verbunden sind. Klinische Studien bestätigen, dass diese Therapieziele durch die Gabe von Epoetin beta erreicht werden. Auch die Wirksamkeit und Sicherheit der einmal wöchentlichen Therapie mit Epoetin beta konnte in aktuellen Studien belegt werden.
Fazit: Epoetin beta erhöht den Hämoglobinwert bei onkologischen Patienten mit Chemotherapie-bedingter Anämie effektiv und schnell. Dies verbessert alle Körperfunktionen, steigert die Lebensqualität der Patienten und vermindert die Transfusionshäufigkeit. Die hohe Wirksamkeit und das günstige Sicherheitsprofil der einmal wöchentlichen Gabe von 30000 IE Epoetin beta wird durch aktuelle Studien und eine große Metaanalyse bestätigt.

Weitere Informationen bei

Roche Pharma AG

Dr. H.-U. Jelitto

Emil-Barell-Straße 1

79639 Grenzach-Wyhlen

Tel. +49 7624 14-2400, Fax -3366

www.roche.de

\title{
Ticker+++ Ticker+++ Ticker+++ Ticker+++ Ticker+++ Ticker+++ Ticker+++
}

Blutspendedienst des Bayerischen Roten Kreuzes (BSD). Der BSD hat in 2 Aktionswochen das Diabetesrisiko bei 10000 Blutspendern bestimmt und dazu ein neues Verfahren getestet, das künftig eine Früherkennung dieser Krankheit ermöglichen könnte. Neben einem Fragebogen zu den Lebensgewohnheiten wurde der $\mathrm{HbA}_{1 \mathrm{c}^{-}}$ Wert gemessen. Dabei hat sich gezeigt, dass erhöhte $\mathrm{HbA}_{1 \mathrm{c}}$-Werte mit bekannten Diabetesrisikofaktoren korrelieren.

Weitere Informationen bei

Blutspendedienst des Bayerischen Roten Kreuzes Andrea Reischl

reischl@lgst.brk.de
Wyeth Pharma. In der Informationsflut des Internets den Überblick zu behalten, ist keine leichte Aufgabe. Im Bereich Nierentransplantation schafft die neudesignte Webseite www.rapamune.de / www.neue-niere.de Klarheit: Eine verbesserte Navigation und ein übersichtlich gestaltetes Design ermöglichen Ärzten (für Fachkreise über DocCheck-Log-In) und Patienten einen noch schnelleren Zugriff auf alle Informationen rund um das Thema Nierentransplantation.

Weitere Informationen bei Wyeth Pharma GmbH Frau Ulrike Schievelbein SchievU@wyeth.com
GlaxoSmithKline (GSK). Im Rahmen des 12. Kongresses der European Hematology Association hat GSK Ergebnisse einer Phase-III-Studie zu dem Wirkstoff Eltrombopag vorgestellt: Der als Tablette eingenommene Thrombopoetin-RezeptorAgonist führte bei adulten Patienten mit chronisch idiopathischer Thrombozytopenie in einer Dosierung von 50 und $75 \mathrm{mg} 1 \times$ täglich zu einem statistisch signifikanten Anstieg der Thrombozytenzahlen und einem verminderten Blutungsrisiko.

Weitere Informationen bei

GlaxoSmithKline GmbH \& Co. KG Arnd Prilipp

arnd.prilipp@gsk.com 